\title{
Hidden heavy flavour production in heavy-ion collisions
}

\author{
Javier Castillo Castellanos ${ }^{1, \star}$ \\ ${ }^{1}$ IRFU, CEA, Université Paris-Saclay, F-91191Gif-sur-Yvette, France
}

\begin{abstract}
An overview of recent experimental results on quarkonium production in heavy-ion collisions at RHIC and LHC energies is presented. Their implications in the theoretical understanding of the production of quarkonia is discussed.
\end{abstract}

\section{Introduction}

Heavy quarks, charm (c) and beauty (b), are important probes of the Quark-Gluon Plasma (QGP). Because of their large mass, heavy-quark pairs are produced by the primary hard collisions of the incoming partons. A small fraction of those initial pairs will bind together to form a quarkonium. Heavy quarks and quarkonia will interact with the QGP throughout its evolution and probe its properties.

Originally, it was proposed that the production of $\mathrm{J} / \psi$ mesons will be suppressed in the QGP by a color-screening mechanism [1]. Then, this was extended to all quarkonium states leading to the sequential suppression scenario: a hierarchy of suppression depending on the binding energy of the quarkonia [2]. Other dynamical mechanisms such as gluon dissociation [3] and quasi-free scattering $[4,5]$ were also proposed. The sequential suppression could inform about the QGP temperature.

More recently, new mechanisms of quarkonium production by the QGP have been proposed. In the statistical hadronization model [6] all heavy quarks and antiquarks, produced in the initial hardscatterings, thermalize in the QGP. Then, all quarkonia are produced by statistical hadronization at the phase boundary of the QGP. Here full suppression of the initially produced quarkonia is assumed. In [5] the approach is extended to consider partial charmonium suppression. Additional quarkonia could also be regenerated throughout the QGP evolution by kinetic recombination of heavy-quarks [7]. In this type of models, called transport models, continuous dissociation and regeneration of quarkonia throughout the life time of the QGP is implemented by a kinetic rate equation. The QGP evolution is described by a hydrodynamical-like expansion. For more recent implementations and calculations of the transport model see [8-10] and [4, 11-13]. These additional production mechanism will therefore compete with the initial quarkonium production and their suppression by the hot medium. The higher initial temperatures of the QGP and the larger heavy-quark production cross sections reached at LHC energies should favor the regeneration mechanism.

Cold nuclear matter (CNM) effects also affect the production of quarkonia in heavy-ion collisions. These can be separated into initial and final-state effects. Initial-state effects occur prior to the formation of the heavy-quark pair, including the modification of the kinematical distribution of the partons in the nuclei (nPDF) compared to that in free nucleons [14-16] and parton energy loss [17, 18]. Final-state effects are those that affect the heavy-quark pair during the finite time it needs to form a

\footnotetext{
^e-mail: Javier.Castillo@cea.fr
} 

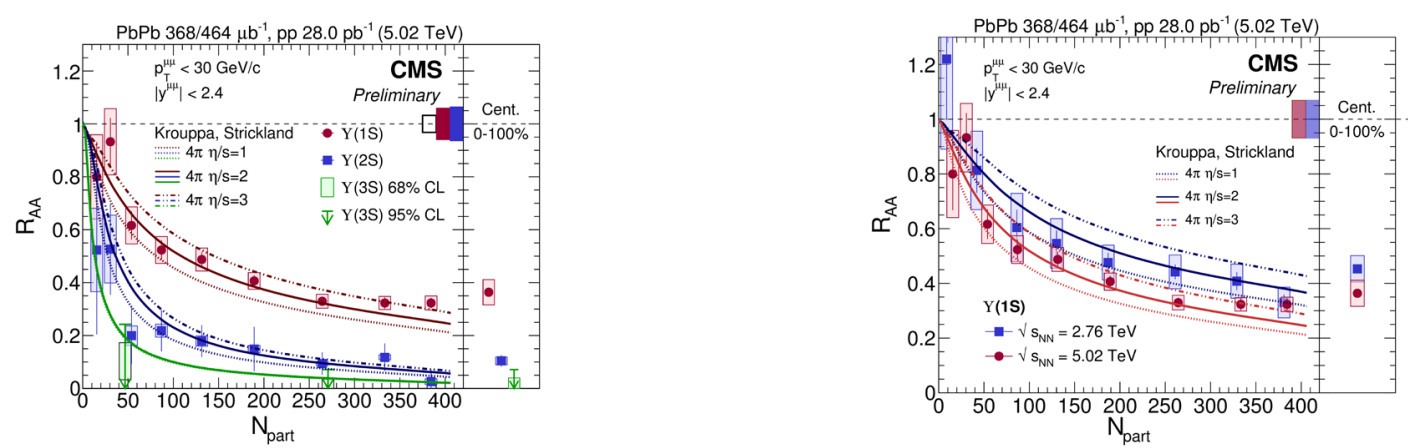

Figure 1. (Left) $R_{\mathrm{AA}}$ of $\Upsilon(\mathrm{nS})$ versus $N_{\text {part }}$ in $\mathrm{Pb}-\mathrm{Pb}$ collisions at $\sqrt{s_{\mathrm{NN}}}=5.02$. (Right) $R_{\mathrm{AA}}$ of $\Upsilon(1 \mathrm{~S})$ versus $N_{\text {part }}$ in $\mathrm{Pb}-\mathrm{Pb}$ collisions at $\sqrt{s_{\mathrm{NN}}}=2.76$ and $5.02 \mathrm{TeV}$. The measurements by CMS [22, 23] are compared to the calculations of a hydrodynamical model $[24,25]$.

quarkonium state or after the state has been formed [19], such as the $Q \bar{Q}$ pair break up by the nuclear matter (expected to be small at high energy) $[19,20]$, and the final-state resonance interaction with surrounding comovers [21]. Finally, parton energy loss could be coherent and thus not factorized into initial and final state effects [18]. Cold nuclear matter effects can be studied in proton-nucleus (p-A) or deuteron-nucleus (d-A) collisions, where the QGP is not expected to be formed.

We will review recent measurements of quarkonium production and their implications in terms of suppression or regeneration mechanisms in the QGP, or CNM effects.

\section{Sequential suppression}

In the sequential suppression scenario, the suppression of a given quarkonium state should increase with increasing temperature or energy density of the QGP. Furthermore, the suppression should increase with decreasing binding energy of the quarkonium state, e.g. the exited $\Upsilon(3 S)$ will be more suppressed than the $\Upsilon(2 \mathrm{~S})$ than the ground $\Upsilon(1 \mathrm{~S})$.

In Fig. 1 (left), the recent measurement by the CMS Collaboration of the nuclear modification factor $R_{\mathrm{AA}}$ of $\Upsilon(\mathrm{nS})$ in $\mathrm{Pb}-\mathrm{Pb}$ at $\sqrt{s_{\mathrm{NN}}}=5.02 \mathrm{TeV}$ is shown versus the collisions centrality, quantified by the number of participating nucleons $N_{\text {part }}$. First, one clearly observes that the $R_{\mathrm{AA}}$ of $\Upsilon(1 \mathrm{~S})$ and $\Upsilon(2 S)$ decrease from peripheral to central collisions, showing that the suppression of $\Upsilon$ is larger in central collisions where the QGP energy density is maximum. Second, for the most central collisions, the $\Upsilon(3 S)$ appears to be more suppressed (or fully suppressed) than the $\Upsilon(2 S)$, which in turn is more suppressed than the $\Upsilon(1 \mathrm{~S})$, confirming the expectations. The comparison of the $\Upsilon(1 \mathrm{~S}) R_{\mathrm{AA}}$ in $\mathrm{Pb}-\mathrm{Pb}$ collisions at $\sqrt{s_{\mathrm{NN}}}=2.76 \mathrm{TeV}$ [22] and 5.02 TeV [23] is shown in Fig. 1 (right). A hint of a stronger $\Upsilon(1 \mathrm{~S})$ suppression at the highest energy can be seen. The behaviors of the $\Upsilon(\mathrm{nS})$ versus the $\Upsilon$ state, the collision centrality and energy are naively expected by the scenarios in which the suppression is solely driven by the QGP energy density. These $\Upsilon(\mathrm{nS})$ measurements are well reproduced by a hydrodynamical model $[24,25]$. In this model, the suppression of the bottomonium states by the QGP, described by a $3+1 \mathrm{~d}$ hydrodynamical model, is based on a complex potential.

Figure 2 (right) shows the STAR measurement of the ratio of the yields of $\Upsilon(2 \mathrm{~S}+3 \mathrm{~S})$ divided by those of $\Upsilon(1 \mathrm{~S})$ versus $N_{\text {part }}$ in $\mathrm{Au}-\mathrm{Au}$ collisions at $\sqrt{s_{\mathrm{NN}}}=0.2 \mathrm{TeV}$ [26]. The horizontal band shows the average of the same ratio for pp collisions. As it was the case at LHC energies, at RHIC energies the excited states of the $\Upsilon$ appear to be more suppressed than ground $\Upsilon(1 \mathrm{~S})$ state. 
The $\Upsilon(1 \mathrm{~S}) R_{\mathrm{AA}}$ measured by ALICE at forward rapidity in $\mathrm{Pb}-\mathrm{Pb}$ collisions at $\sqrt{s_{\mathrm{NN}}}=5.02$ $\mathrm{TeV}$ [27] is shown in Fig. 2 as a function of rapidity together with the measurement by CMS. The two experiments complement each other and cover a wide rapidity range $(0<y<4)$. The experimental measurements are compared to the calculations of the hydrodynamical model [25]. The model is able to reproduce the $\Upsilon(1 \mathrm{~S}) R_{\mathrm{AA}}$ over the full rapidity range, although at forward $y$ the trends of the model and the ALICE $R_{\mathrm{AA}}$ seem opposite. Such different trend was more evident at the lower LHC energy (see e.g. [28]). In this context, a more precise measurement at forward $y$ will be important. Indeed, in the hydrodynamical model [25] only suppression of bottomonia by the QGP is considered, neglecting CNM effects and any possible regeneration component. Transport models [12, 29], which include, in addition to a suppression by the QGP, CNM effects and a small regeneration component obtain an inverted rapidity dependence for the $\Upsilon(1 \mathrm{~S}) R_{\mathrm{AA}}$. Finally, to answer the question if direct $\Upsilon(1 \mathrm{~S})$ are suppressed, a precise understanding of the CNM effects is needed.

\section{Regeneration}

Due to the large initial charm-quark density, the charmonium family is best suited to investigate any possible quarkonium regeneration mechanism. Figure 3 shows the recent $\mathrm{J} / \psi R_{\mathrm{AA}}$ at forward (left) and mid- (right) $y$ versus $N_{\text {part }}$ measured by ALICE in Pb-Pb collisions at $\sqrt{s_{\mathrm{NN}}}=5.02 \mathrm{TeV}[31,32]$, compared to the earlier measurements at $\sqrt{s_{\mathrm{NN}}}=2.76 \mathrm{TeV}[30,33,34]$ and in $\mathrm{Au}-\mathrm{Au}$ collisions at $\sqrt{s_{\mathrm{NN}}}=0.2 \mathrm{TeV}$ by PHENIX $[35,36]$. At RHIC energies, the $\mathrm{J} / \psi R_{\mathrm{AA}}$ exhibits a decreasing trend from peripheral to central collisions, whereas at LHC energies a saturation of the $R_{\mathrm{AA}}$ is seen for $N_{\text {part }} \gtrsim 100$. At mid- $y$ even a hint of an increased $R_{\mathrm{AA}}$ for the most central collisions is seen. For the most central collisions, the $\mathrm{J} / \psi R_{\mathrm{AA}}$ is larger at LHC than at RHIC energies, i.e. the $\mathrm{J} / \psi$ suppression is smaller where the QGP energy density is higher. Such observations clearly establish the case for an important contribution of the regeneration mechanism at the LHC. Models implementing full [37] or partial $[10,11,13,21,38,39] \mathrm{J} / \psi$ regeneration are able to reproduce these results.

The regeneration of $\mathbf{J} / \psi$ is expected to contribute mostly at low $p_{\mathrm{T}}$. Figure 4 (left) shows the $\mathrm{J} / \psi$ $R_{\mathrm{AA}}$ at forward $y$ versus $p_{\mathrm{T}}$ in $\mathrm{Pb}-\mathrm{Pb}$ collisions at $\sqrt{s_{\mathrm{NN}}}=5.02 \mathrm{TeV}$ for three centrality classes [40]. The bottom panel shows the ratio of the $R_{\mathrm{AA}}$ at $\sqrt{s_{\mathrm{NN}}}=5.02$ and $2.76 \mathrm{TeV}$. For most central collisions, the $R_{\mathrm{AA}}$ increases with decreasing $p_{\mathrm{T}}$ confirming the contribution of $\mathrm{J} / \psi$ regeneration at low $p_{\mathrm{T}}$. The calculations of a transport model $[11,13,38]$ including $\mathrm{J} / \psi$ regeneration can reproduce the
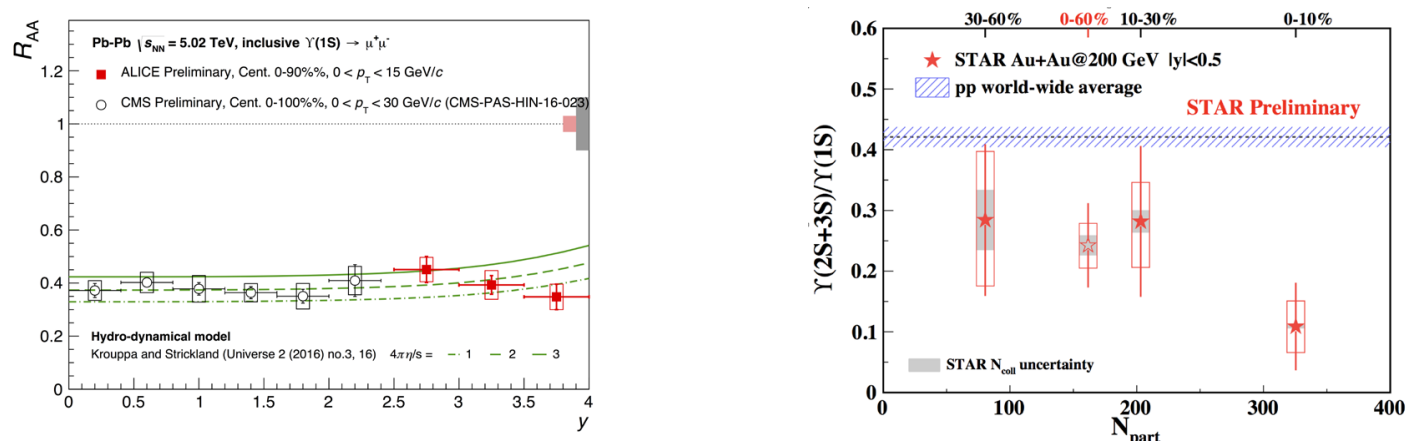

Figure 2. (Left) $R_{\mathrm{AA}}$ of $\Upsilon(1 \mathrm{~S})$ versus rapidity in $\mathrm{Pb}-\mathrm{Pb}$ collisions at $\sqrt{s_{\mathrm{NN}}}=5.02 \mathrm{TeV}[23,27]$ compared to the calculations of a hydrodynamical model [25]. (Right) Ratio of yields of $\Upsilon(2 \mathrm{~S}+3 \mathrm{~S})$ over $\Upsilon(1 \mathrm{~S})$ in $\mathrm{Au}-\mathrm{Au}$ collisions at $\sqrt{s_{\mathrm{NN}}}=0.2 \mathrm{TeV}[26]$ compared to the same ratio in pp collisions shown as a band. 

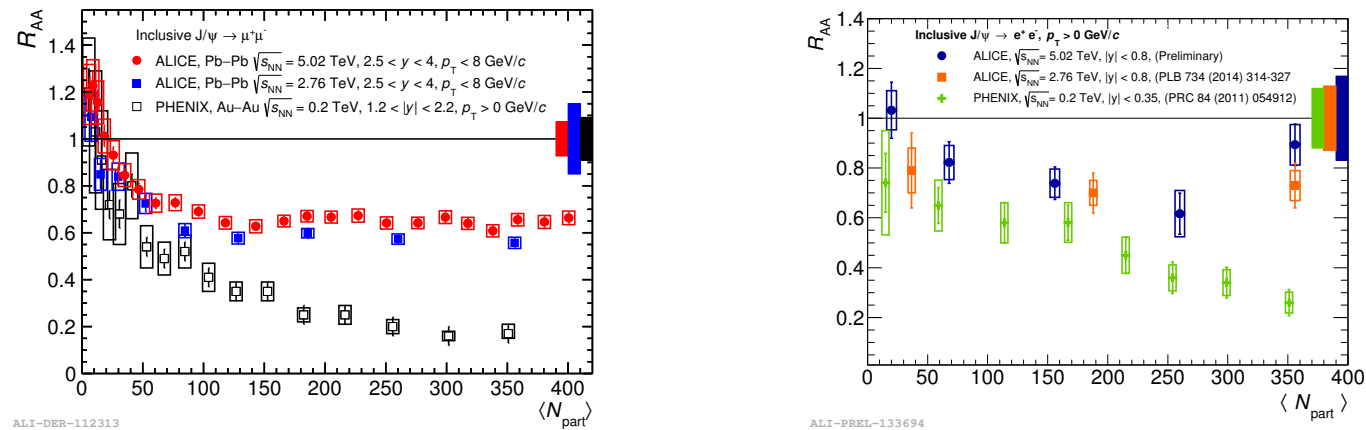

Figure 3. $\mathrm{J} / \psi R_{\mathrm{AA}}$ at forward (left) and mid- (right) $y$ versus $N_{\text {part }}$ in $\mathrm{Pb}-\mathrm{Pb}$ collisions at $\sqrt{s_{\mathrm{NN}}}=5.02 \mathrm{TeV}$ and $\sqrt{s_{\mathrm{NN}}}=2.76 \mathrm{TeV}[30-34]$ and in $\mathrm{Au}-\mathrm{Au}$ collisions at $\sqrt{s_{\mathrm{NN}}}=0.2 \mathrm{TeV}[35,36]$.

observed trends. However, given the much improved uncertainties of the experimental measurements, quantitative tensions are observed. The new $\mathrm{J} / \psi R_{\mathrm{AA}}$ vs. $p_{\mathrm{T}}$ at mid- $y$ [32] is compared with the one at forward $y$ [31] in Fig. 4 (right). Although the uncertainties of the mid-y $R_{\mathrm{AA}}$ are still large, one can appreciate at low $p_{\mathrm{T}}$ a larger $R_{\mathrm{AA}}$ at mid- than at forward $y$, which would indicate a larger contribution of $\mathrm{J} / \psi$ regeneration where the QGP energy density and the charm-quark density are larger.

Thermalized charm quarks will be part of the collective motion of the QGP and acquire flow, in particular elliptic flow. Regenerated $\mathrm{J} / \psi$ produced by the recombination of thermalized charm quarks will therefore inherit their elliptic flow, resulting in a large $v_{2}$. The new measurements by the ALICE Collaboration of the $\mathrm{J} / \psi v_{2}$ at forward $y$ in $\mathrm{Pb}-\mathrm{Pb}$ collisions at $\sqrt{s_{\mathrm{NN}}}=5.02 \mathrm{TeV}$ [44] establish, for the first time, an unambiguous non-zero $v_{2}$ for $\mathrm{J} / \psi$. Figure 5 shows the $\mathrm{J} / \psi v_{2}$ versus $p_{\mathrm{T}}$ in three classes of centrality, $5-20 \%, 20-40 \%$, and $40-60 \%$. The $\mathrm{J} / \psi v_{2}$ increases with $p_{\mathrm{T}}$ up to $p_{\mathrm{T}} \sim 6 \mathrm{GeV} / \mathrm{c}$ and decreases or saturates thereafter. The $v_{2}$ also appears to be (marginally) maximum in the 20-40\% centrality class, in contrast to light hadrons [45]. The $v_{2}$ of D-mesons at mid- $y$ and in a somewhat different centrality class (30-50\%) [41] is also shown. Clearly, both $\mathrm{D}$ and $\mathrm{J} / \psi$ mesons show a positive $v_{2}$ in the explored $p_{\mathrm{T}}$ region, with the $\mathrm{J} / \psi v_{2}$ being lower than the $\mathrm{D}$-meson at low and intermediate $p_{\mathrm{T}}$. Such observation strongly supports the scenario in which both $\mathrm{D}$ and $\mathrm{J} / \psi$ mesons inherit the $v_{2}$
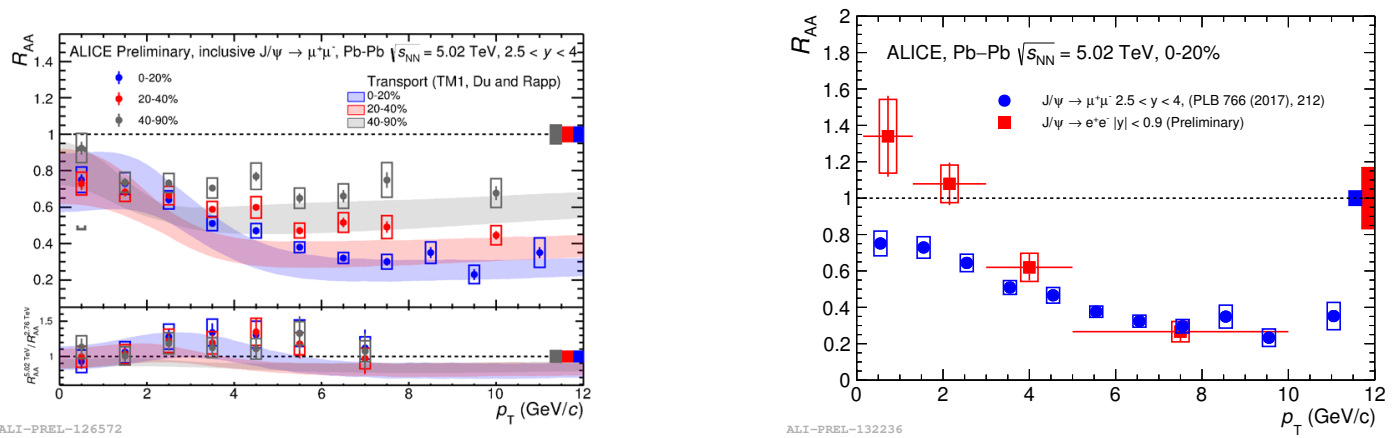

Figure 4. $R_{\mathrm{AA}}$ of $\mathrm{J} / \psi$ versus $p_{\mathrm{T}}$ in $\mathrm{Pb}-\mathrm{Pb}$ collisions at $\sqrt{s_{\mathrm{NN}}}=5.02 \mathrm{TeV}$ : (left) at forward $y$ in three centrality classes [40] and (right) at forward and mid-y in central collisions [31, 32]. 

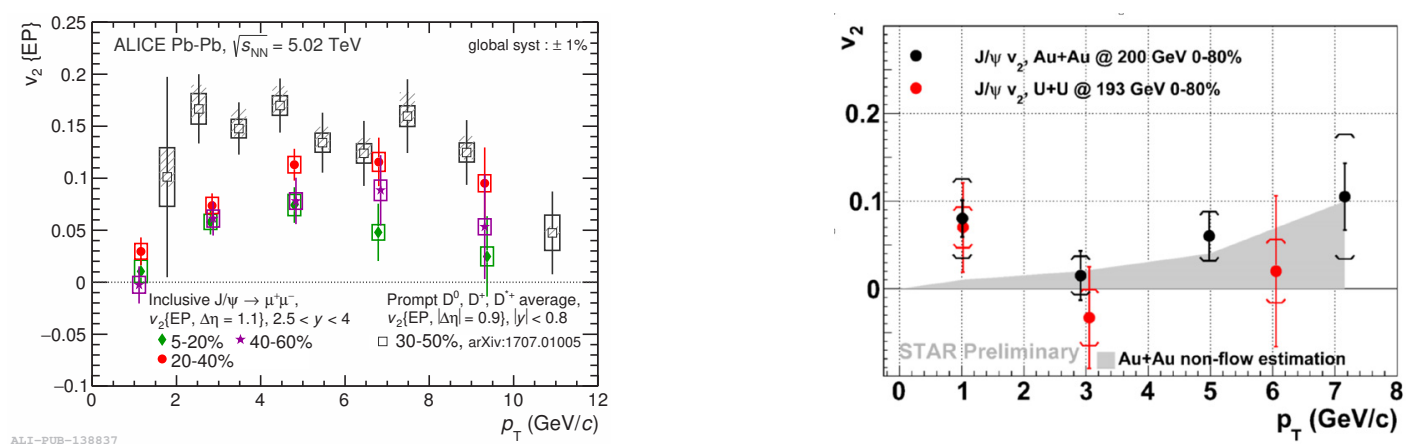

Figure 5. (Left) Inclusive $\mathrm{J} / \psi v_{2}\left(p_{\mathrm{T}}\right)$ at forward $y$ in $\mathrm{Pb}-\mathrm{Pb}$ collisions at $\sqrt{s_{\mathrm{NN}}}=5.02 \mathrm{TeV}$ for three centrality classes. The D-meson $v_{2}\left(p_{\mathrm{T}}\right)$ at mid-y in the centrality class 30-50\% is shown for comparison [41]. (Right) Inclusive $\mathrm{J} / \psi v_{2}\left(p_{\mathrm{T}}\right)$ in $\mathrm{Au}-\mathrm{Au}$ and $\mathrm{U}-\mathrm{U}$ collisions at $\sqrt{s_{\mathrm{NN}}}=0.2$ and $0.193 \mathrm{TeV}$, respectively [42, 43].
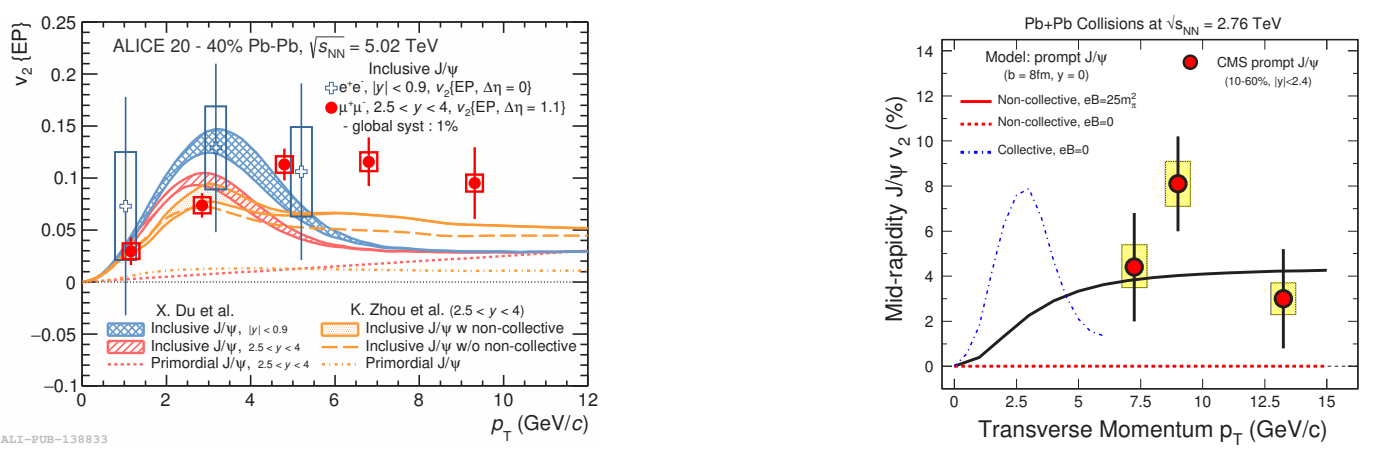

Figure 6. (Left) Inclusive $\mathrm{J} / \psi v_{2}\left(p_{\mathrm{T}}\right)$ in semi-central (20-40\%) $\mathrm{Pb}-\mathrm{Pb}$ collisions at $\sqrt{s_{\mathrm{NN}}}=5.02 \mathrm{TeV}$ [44] compared to transport model calculations by [38] and [10]. (Right) Prompt $\mathrm{J} / \psi v_{2}\left(p_{\mathrm{T}}\right)$ in $10-60 \%$ non-central $\mathrm{Pb}-\mathrm{Pb}$ collisions at $\sqrt{s_{\mathrm{NN}}}=2.76 \mathrm{TeV}$ [46] compared to transport model calculations [10, 47].

from thermalized charm quarks. We note that at RHIC energies, where the regeneration contribution was found to be small, the measured $\mathrm{J} / \psi v_{2}$ is compatible with zero in $\mathrm{Au}-\mathrm{Au}$ and $\mathrm{U}-\mathrm{U}$ collisions at $\sqrt{s_{\mathrm{NN}}}=0.2$ and $0.193 \mathrm{TeV}$, respectively, as measured by STAR [42, 43] (see Fig. 5 (right)).

Figure 6 (left) shows the $\mathrm{J} / \psi v_{2}\left(p_{\mathrm{T}}\right)$ at forward and mid- $y$ in semi-central $\mathrm{Pb}-\mathrm{Pb}$ collisions at $\sqrt{s_{\mathrm{NN}}}=5.02 \mathrm{TeV}$ compared to the calculations of two transport models. The model by Du et al. [38] reproduces the magnitude of the $\mathrm{J} / \psi v_{2}$ at forward $y$ and low $p_{\mathrm{T}}$ by a dominant contribution of $\mathrm{J} / \psi$ elliptic flow inherited from thermalized charm quarks. However, the overall shape of the $v_{2}\left(p_{\mathrm{T}}\right)$ is missed and the $v_{2}$ at high $p_{\mathrm{T}}$ is underestimated. This disagreement suggests a missing mechanism in the model. Similar conclusions can be drawn from the comparison to the model by Zhou et al. [10]. The authors add a $v_{2}$ arising from a possible strong magnetic field in the early stage of heavy-ion collisions [47], which improves the comparison with the measurement, especially at high $p_{\mathrm{T}}$. Such non-collective component [47] was able to reproduce the prompt $\mathrm{J} / \psi v_{2}$ at high $p_{\mathrm{T}}$ measured by CMS in $\mathrm{Pb}-\mathrm{Pb}$ collisions at $\sqrt{s_{\mathrm{NN}}}=2.76 \mathrm{TeV}$ [46] (see Fig. 6 (right)).

We note that some tension is also seen between the transport model calculations and the $\mathrm{J} / \psi R_{\mathrm{AA}}$ measurements in the intermediate region $4<p_{\mathrm{T}}<8 \mathrm{GeV} / \mathrm{c}$. Clearly, the improved precision of both 

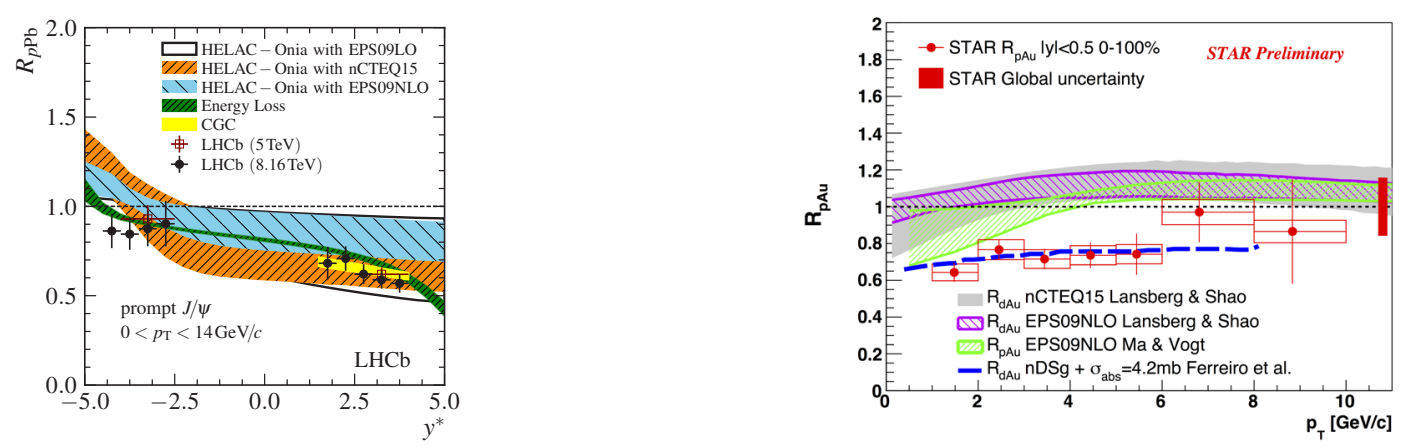

Figure 7. (Left) $R_{\mathrm{pPb}}$ of prompt $\mathrm{J} / \psi$ versus rapidity in $\mathrm{p}-\mathrm{Pb}$ collisions at $\sqrt{s_{\mathrm{NN}}}=8.16 \mathrm{TeV}[52]$. (Right) $\mathrm{J} / \psi R_{\mathrm{pAu}}$ at $\sqrt{s_{\mathrm{NN}}}=0.2 \mathrm{TeV}[43]$ compared to various model calculations $[48,50,51]$.

$R_{\mathrm{AA}}$ and $v_{2}$ measurements put strong constraints in the production models and will help to further improve our understanding of the $\mathrm{J} / \psi$ production mechanism in heavy-ion collisions.

\section{Cold Nuclear Matter effects}

In addition to the suppression and regeneration in the QGP, CNM effects also affect the production of quarkonia in heavy-ion collisions. Also here the recent improvement of the measurement uncertainties are seriously challenging the theoretical calculations.

The prompt $\mathrm{J} / \psi R_{\mathrm{pPb}}$ measured by LHCb in $\mathrm{p}-\mathrm{Pb}$ collisions at $\sqrt{s_{\mathrm{NN}}}=8.16 \mathrm{TeV}$ is shown in Fig. 7 (left) versus rapidity. The results in the p-going $(y>0)$ and Pb-going $(y<0)$ directions are compared to model calculations including shadowing [48], parton energy loss [18], or CGC [49]. While the models are able to qualitatively describe the measured $R_{\mathrm{pPb}}$, the quantitative agreement is not satisfactory. Figure 7 (right) shows the new $\mathrm{J} / \psi R_{\mathrm{pAu}}$ measured by STAR at $\sqrt{s_{\mathrm{NN}}}=0.2 \mathrm{TeV}$ compared to model calculations $[48,50,51]$. At RHIC energies, the inclusion of a nuclear absorption cross section $\sigma_{\mathrm{abs}}=4.2 \mathrm{mb}^{-1}$ [51] is necessary to describe the $R_{\mathrm{pAu}}$.

In the bottomonium sector, we highlight the new measurement by ATLAS of the $\Upsilon(1 \mathrm{~S}) R_{\mathrm{pPb}}$ at mid- $y$ in $\mathrm{p}-\mathrm{Pb}$ collisions at $\sqrt{s_{\mathrm{NN}}}=5.02 \mathrm{TeV}$, which nicely complement the earlier measurements by ALICE [53] and LHCb [54], shown in Fig. 8 (left). A small suppression of the $\Upsilon(1 \mathrm{~S})$ is seen at mid- $y$, which could be explained by shadowing (plus comover interactions, as shown in the figure) or parton energy loss (see e.g. [53]). The improvement of the measurements in the backward region will be important to distinguish between nPDF parameterizations with large or small gluon anti-shadowing.

Finally, among the striking results from the p-A programs at RHIC and LHC, we mention the observation of a stronger suppression of the excited states compared to the ground state. Measurements of the production of $\mathrm{J} / \psi$ and $\psi(2 \mathrm{~S})$ at forward and backward $y$ in $\mathrm{p}-\mathrm{Pb}$ collisions at $\sqrt{s_{\mathrm{NN}}}=5.02$ $\mathrm{TeV}$ have shown that the $\psi(2 \mathrm{~S})$ is more suppressed than the $\mathrm{J} / \psi$, that this difference is stronger in the $\mathrm{Pb}$-going than in the p-going direction, and that this difference increases with the centrality of the collision [55]. The new measurement by CMS at mid- $y$ shown in Fig. 8 (right) confirms such behavior. The $R_{\mathrm{pPb}}$ of the $\psi(2 \mathrm{~S})$ is smaller than that of the $\mathrm{J} / \psi$, and the difference increases from positive to negative rapidity. The CMS and ATLAS collaborations have also observed a stronger suppression of the $\Upsilon(2 \mathrm{~S})$ compared to the $\Upsilon(1 \mathrm{~S})$ at mid- $y$ in $\mathrm{p}-\mathrm{Pb}$ collisions at $\sqrt{s_{\mathrm{NN}}}=5.02 \mathrm{TeV}[56,57]$. The observed difference is currently reproduced only by theoretical models including, in addition to initial-state effects, some final-state interaction with the comoving hadronic medium [21, 38]. 

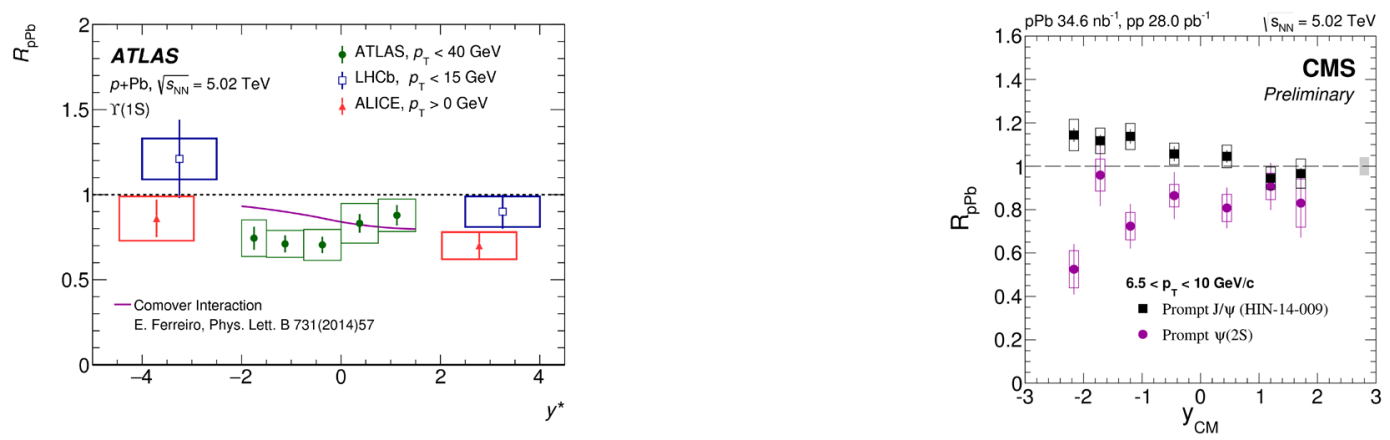

Figure 8. (Left) $R_{\mathrm{pPb}}$ of $\Upsilon(1 \mathrm{~S})$ versus rapidity in $\mathrm{p}-\mathrm{Pb}$ collisions at $\sqrt{s_{\mathrm{NN}}}=5.02 \mathrm{TeV}$ measured by ALICE [53], ATLAS [57] and LHCb [54]. (Right) $R_{\mathrm{pPb}}$ of $\mathrm{J} / \psi$ and $\psi(2 \mathrm{~S})$ at mid- $y$ measured by CMS [58, 59].

\section{Summary}

In summary, the field of quarkonium production in heavy-ion collisions is a very dynamic and prolific one. In the last years the following picture has emerged. The production of quarkonia is suppressed in the QGP, with the loosely bound states being more suppressed than the tightly bound ones. The sequential suppression is evident in the bottomonium sector at both RHIC and LHC energies and for high $p_{\mathrm{T}} \mathrm{J} / \psi$. At high energies, new quarkonia are produced by statistical hadronization or regeneration from thermalized charm quarks in the QGP. Such regeneration mechanism is mostly seen for low $p_{\mathrm{T}}$ $\mathrm{J} / \psi$ at the LHC. While this picture is supported by numerous measurements, the high quality of the new results are challenging the available models and calling for a coherent description of all of them.

\section{References}

[1] T. Matsui, H. Satz, Phys. Lett. B178, $416(1986)$

[2] F. Karsch, M.T. Mehr, H. Satz, Z. Phys. C37, 617 (1988)

[3] Y. Park, K.I. Kim, T. Song, S.H. Lee, C.Y. Wong, Phys. Rev. C76, 044907 (2007), 0704.3770

[4] X. Zhao, R. Rapp, Phys. Lett. B664, 253 (2008), 0712 . 2407

[5] L. Grandchamp, R. Rapp, Phys. Lett. B523, 60 (2001), hep-ph/0103124

[6] P. Braun-Munzinger, J. Stachel, Phys. Lett. B490, 196 (2000), nucl-th/0007059

[7] R.L. Thews, M. Schroedter, J. Rafelski, Phys. Rev. C63, 054905 (2001), hep-ph/0007323

[8] L. Yan, P. Zhuang, N. Xu, Phys. Rev. Lett. 97, 232301 (2006), nucl-th/0608010

[9] Y. Liu, Z. Qu, N. Xu, P. Zhuang, Phys. Lett. B678, 72 (2009), 0901. 2757

[10] K. Zhou, N. Xu, Z. Xu, P. Zhuang, Phys. Rev. C89, 054911 (2014), 1401.5845

[11] X. Zhao, R. Rapp, Nucl. Phys. A859, 114 (2011), 1102.2194

[12] A. Emerick, X. Zhao, R. Rapp, Eur. Phys. J. A48, 72 (2012), 1111.6537

[13] X. Du, R. Rapp, J. Phys. Conf. Ser. 779, 012042 (2017), 1609.04868

[14] K.J. Eskola, H. Paukkunen, C.A. Salgado, JHEP 04, 065 (2009), 0902.4154

[15] D. de Florian, R. Sassot, P. Zurita, M. Stratmann, Phys. Rev. D85, 074028 (2012), 1112.6324

[16] M. Hirai, S. Kumano, T.H. Nagai, Phys. Rev. C76, 065207 (2007), 0709. 3038

[17] S. Gavin, J. Milana, Phys. Rev. Lett. 68, 1834 (1992)

[18] F. Arleo, S. Peigne, JHEP 03, 122 (2013), 1212 . 0434 
[19] R. Vogt, Phys. Rev. C81, 044903 (2010), 1003. 3497

[20] E.G. Ferreiro, F. Fleuret, J.P. Lansberg, N. Matagne, A. Rakotozafindrabe, Eur. Phys. J. C73, 2427 (2013), 1110.5047

[21] E.G. Ferreiro, Phys. Lett. B749, 98 (2015), 1411.0549

[22] V. Khachatryan et al. (CMS), Phys. Lett. B770, 357 (2017), 1611.01510

[23] CMS (CMS), CMS-PAS-HIN-16-023 (2017)

[24] B. Krouppa, R. Ryblewski, M. Strickland, Phys. Rev. C92, 061901 (2015), 1507.03951

[25] B. Krouppa, M. Strickland, Universe 2, 16 (2016), 1605.03561

[26] Z. Ye (STAR), Nucl. Phys. A967, 600 (2017)

[27] A. Francisco (ALICE), These proceedings (2017)

[28] B.B. Abelev et al. (ALICE), Phys. Lett. B738, 361 (2014), 1405 . 4493

[29] K. Zhou, N. Xu, P. Zhuang, Nucl. Phys. A931, 654 (2014), 1408. 3900

[30] B. Abelev et al. (ALICE), Phys. Rev. Lett. 109, 072301 (2012), 1202.1383

[31] J. Adam et al. (ALICE), Phys. Lett. B766, 212 (2017), 1606. 08197

[32] R.T. Jiménez Bustamante (ALICE), Nucl. Phys. A967, 576 (2017)

[33] J. Adam et al. (ALICE), JHEP 05, 179 (2016), 1506.08804

[34] J. Adam et al. (ALICE), JHEP 07, 051 (2015), 1504.07151

[35] A. Adare et al. (PHENIX), Phys. Rev. C84, 054912 (2011), 1103. 6269

[36] A. Adare et al. (PHENIX), Phys. Rev. Lett. 98, 232301 (2007), nucl-ex/0611020

[37] A. Andronic, P. Braun-Munzinger, K. Redlich, J. Stachel, Nucl. Phys. A904-905, 535c (2013), 1210.7724

[38] X. Du, R. Rapp, Nucl. Phys. A943, 147 (2015), 1504 . 00670

[39] E.G. Ferreiro, Phys. Lett. B731, 57 (2014), 1210. 3209

[40] M. Tarhini (ALICE), Nucl. Phys. A967, 588 (2017)

[41] S. Acharya et al. (ALICE) (2017), 1707.01005

[42] L. Adamczyk et al. (STAR), Phys. Rev. Lett. 111, 052301 (2013), 1212 . 3304

[43] X. Huang (STAR), These proceedings (2017)

[44] S. Acharya et al. (ALICE) (2017), 1709.05260

[45] B.B. Abelev et al. (ALICE), JHEP 06, 190 (2015), 1405.4632

[46] V. Khachatryan et al. (CMS), Eur. Phys. J. C77, 252 (2017), 1610. 00613

[47] X. Guo, S. Shi, N. Xu, Z. Xu, P. Zhuang, Phys. Lett. B751, 215 (2015), 1502.04407

[48] H.S. Shao, Comput. Phys. Commun. 198, 238 (2016), 1507.03435

[49] B. Ducloué, T. Lappi, H. Mäntysaari, Phys. Rev. D94, 074031 (2016), 1605.05680

[50] J.P. Lansberg, H.S. Shao, Eur. Phys. J. C77, 1 (2017), 1610. 05382

[51] E.G. Ferreiro, F. Fleuret, J.P. Lansberg, N. Matagne, A. Rakotozafindrabe, Few Body Syst. 53, 27 (2012), 1201.5574

[52] R. Aaij et al. (LHCb), Phys. Lett. B774, 159 (2017), 1706.07122

[53] B.B. Abelev et al. (ALICE), Phys. Lett. B740, 105 (2015), 1410. 2234

[54] R. Aaij et al. (LHCb), JHEP 07, 094 (2014), 1405 . 5152

[55] J. Adam et al. (ALICE), JHEP 06, 050 (2016), 1603.02816

[56] S. Chatrchyan et al. (CMS), JHEP 04, 103 (2014), 1312.6300

[57] M. Aaboud et al. (ATLAS) (2017), 1709.03089

[58] A.M. Sirunyan et al. (CMS), Eur. Phys. J. C77, 269 (2017), 1702.01462

[59] CMS (CMS), CMS-PAS-HIN-16-015 (2017) 